\title{
Response Time Bounds for Sporadic Arbitrary-Deadline Tasks under Global Fixed-Priority Scheduling on Multiprocessors
}

\author{
Wen-Hung Huang and Jian-Jia Chen \\ Department of Computer Science \\ TU Dortmund University, Germany
}

\begin{abstract}
In this paper, we study the problem of scheduling arbitrarydeadline real-time sporadic task sets on a multiprocessor system under global fixed-priority scheduling. Two contributions are made in this paper. First, it has been shown that the existing response time analysis in arbitrarydeadline systems is flawed: the response time may be larger than the derived bound. This paper provides a revised analysis resolving the problems with the original approach, and then propose a corresponding schedulability test. Secondly, we derive a linear-time upper bound on the response time of arbitrary-deadline tasks in multiprocessor systems. To the best of our knowledge, this is the first work presenting a linear-time response time upper bound for arbitrary-deadline sporadic tasks in multiprocessor systems. Empirically, this linear-time response time bound is shown to be highly effective in terms of the number of task sets that are deemed schedulable.
\end{abstract}

\section{INTRODUCTION}

Analyzing the worst-case response time is one of the most important issues for designing hard real-time systems to ensure the timeliness of tasks, especially for safety-critical embedded systems. There have been extensive results in such a general direction since the seminal paper by Liu and Layland [24]. A well-adopted real-time task model is the sporadic task model, in which a sporadic task $\tau_{i}$ defines an infinite sequence of task instances, also called jobs: (1) Any two consecutive jobs of task $\tau_{i}$ should be temporally separated by at least $T_{i}$. (2) The execution time of a job of task $\tau_{i}$ is at most $C_{i}$ (also known as the worst-case execution time). The response time of a job of task $\tau_{i}$ is its completion time minus its arrival time. Therefore, the worst-case response time of task $\tau_{i}$ is (an upper bound of) the maximum response time among all the jobs of task $\tau_{i}$.

Typically, for real-time systems, a sporadic task $\tau_{i}$ also has an associated relative deadline $D_{i}$. To ensure that the relative deadline of task $\tau_{i}$ is met, we essentially have to

Permission to make digital or hard copies of part or all of this work for personal or classroom use is granted without fee provided that copies are not made or distributed for profit or commercial advantage and that copies bear this notice and the full citation on the first page. Copyrights for third-party components of this work must be honored. For all other uses, contact the owner/author(s).

RTNS '15 November 04-06, 2015, Lille, France

(C) 2015 Copyright held by the owner/author(s).

ACM ISBN 978-1-4503-3591-1/15/11.

DOI: http://dx.doi.org/10.1145/2834848.2834849 verify whether the worst-case response time of task $\tau_{i}$ is at most $D_{i}$. Verifying whether a set of sporadic tasks can meet all the relative deadlines by a scheduling algorithm is called a schedulability test. The difficulty of the schedulability tests also depends on the relationship of the relative deadlines and the periods of the tasks, which are typically classified with the following cases: (1) implicit deadlines if the relative deadlines of the sporadic tasks are equal to their minimum inter-arrival times, i.e., $T_{i}=D_{i}, \forall \tau_{i},(2)$ constrained deadlines if the minimum inter-arrival times are no less than their relative deadlines, i.e., $D_{i} \leq T_{i}, \forall \tau_{i}$, and (3) arbitrary deadlines, otherwise. It is evident that the case with arbitrary deadlines is the most general one.

For uniprocessor fixed-priority scheduling in arbitrarydeadline task systems, the exact schedulability tests and the (tight) worst-case response time using time-demand analysis (TDA) are proposed by Lehoczky [23,30]. Such results may suffer from their high time-complexity because of the explosion of busy interval in arbitrary-deadline systems. From the designers' perspective, a fast test is more applicable during design space exploration, even at a price of the accuracy. Several approaches have been proposed for reducing the computational complexity of the TDA $[13,27]$. Lehoczky proposes a utilization upper bound for a set of periodic arbitrary-deadline tasks under fixed-priority scheduling [12]. Bini proposes a quadratic bound by considering some information about the task periods [12]. The linear-time response-time bound for fixed-priority systems has been first proposed by Davis and Burns [17]. This bound has been later improved by Bini et al. [15].

To schedule real-time tasks on multiprocessor platforms, there are three widely adopted paradigms: partitioned, global, and semi-partitioned scheduling [19]. In this paper, we consider global scheduling, in which a job can migrate from one processor to another processor during its executions. But, a job cannot be simultaneously executed on more than one processor.

Unfortunately, deriving exact schedulability tests under multiprocessor global scheduling is extremely harder than in uniprocessor due to the non-existence of critical instant. The first brute force approach regarding the exact response time is proposed by Baker and Cirinei [5]. The analysis consists in solving reachability problem in a finite state machine that represents all possible combinations of arrival times and execution sequences for a task set. In addition, some results are recently proposed to reduce the number of states 215 to be analyzed in the reachability analysis [21,28]. Gen- 
erally speaking, these approaches are so complex that only task sets with very small integer periods can be tractably solved. Alternatively, most of the research in the literature focus on finding approximate upper bounds to the response time. The response time analysis for implicit-deadline sporadic task systems has been studied by Andersson et al. [1]. The fixed-priority scheduling of constrained-deadline sporadic task systems has been studied in the literature $[3,6,11]$.

Regarding arbitrary-deadline task systems, several results have been proposed in the literature $[4,7,22,29]$. Baker [4] designs a test based on some deep insights, in that if a task $\tau_{k}$ misses its deadline, then the load in the analyzed interval, called problem window, must satisfy some necessary conditions on the parameters of all the tasks. Baruah and Fisher [7] use another annotation to extend the analysis window and derive a corresponding pseudo-polynomial-time schedulability test. The first worst-case response-time analysis for arbitrary-deadline task systems is proposed by Guan et al. [22], in which the authors use the insight proposed by Baruah [8] to limit the number of carry-in tasks, and then apply the workload function proposed by Bertogna et al. [11] to quantify the requested demand of higher-priority tasks. Unfortunately, it has recently been shown in [29] that the analysis by Guan et al. [22] is optimistic for arbitrarydeadline tasks. The assumption that each carry-in task has only one carry-in job in [22] is in fact problematic, as a carry-in task can be shown to carry more than one carry-in job [29]. Sun et al. [29] derive a complex carry-in workload function for the response time analysis where all possible combinations of carry-in and non-carry-in functions have to be explicitly enumerated. However, such a method is computationally intractable since the time complexity is exponential.

Contributions. We focus in this paper on providing worstcase response time analyses by using the concept that has been adopted by Guan et al. [22] with proper annotations. Even though the assumption that each carry-in task has only one carry-in job is problematic in [22], we can safely bound the interference from the carry-in task by means of proper definitions of the analysis window and annotations, to be shown in Section 4. We summarize the significance of this work as follows:

- The proper annotation of the number of carry-in jobs of a carry-in task results in a revisited response time analysis, in Section 4. Our analysis is distinct from other analyses $[4,7]$ in that our analysis quantifies the carry-in function more precisely.

- In Section 6, we derive the first known linear-time response time bound on arbitrary-deadline multiprocessor systems. To the best of our knowledge, this is the first work presenting a linear-time response time bound on arbitrary-deadline multiprocessors.

- We evaluate our results in Section 7 by comparing to the state-of-the-art results for both constraineddeadline systems $[3,6,11,22]$ and arbitrary-deadline systems $[4,7]$. The experimental results show that our time-demand analysis is comparable to the state of the art [22] and superior to the others $[3,6,11]$ for constrained-deadline systems. Regarding arbitrarydeadline systems, our TDA is much better than the existing results $[4,7]$. Moreover, compared to the existing analyses $[3,4,6,7,11]$, the proposed lineartime upper bound on the worst-case response time is shown to be highly effective, especially for large ranges of periods, for which the time-demand analysis suffers from its high computational complexity.

\section{SYSTEM MODEL AND PROBLEM SET- TING}

This paper considers a set of $n$ independent arbitrarydeadline sporadic real-time tasks $\tau=\left\{\tau_{1}, \tau_{2}, \ldots, \tau_{n}\right\}$ upon a system comprised of $m$ identical processors, where $m \geq 1$ is an integer. Each task can release an infinite number of jobs (also called task instances) under the given minimum interarrival time (temporal) constraint. We consider the sporadic task model [25], where $C_{i}$ denotes the worst-case execution time (WCET) of task $\tau_{i}$, the minimum inter-arrival time or period $T_{i}$ represents the minimum temporal distance between consecutive arrivals of task instances, and the relative deadline $D_{i}$ represents the response time constraint for executing the job. If a task instance arrives at time $\theta_{a}$, the execution of this instance must be finished no later than its absolute deadline $\theta_{a}+D_{i}$ and the next instance of the task must arrive no earlier than $\theta_{a}$ plus the minimum inter-arrival time, i.e., $\theta_{a}+T_{i}$. We assume that all task parameters are positive integers. Since all the $m$ processors are identical, the execution time of a job of a task does not depend on the processors that execute the job.

Task system $\tau$ is an implicit-deadline system if each task $\tau_{i} \in \tau$ has its relative deadline $D_{i}$ equal to its period $T_{i}$, and a constrained-deadline system if each task $\tau_{i} \in \tau$ has relative deadline $D_{i}$ no larger than its period $T_{i}$. Otherwise, task system $\tau$ is an arbitrary-deadline system. In this paper we consider the most general arbitrary-deadline system.

We assume that the system is fully preemptive, and allows for global inter-processor migration. We assume that the cost of preemption and migration has been subsumed into the worst-case execution time of each task. In this paper each task is assumed to be preemptively scheduled on $m$ identical multiprocessor systems according to a global fixedpriority scheduling, in which each task is associated with a unique priority level. We assume that the jobs of the same task are served by the first-come first-serve (FCFS) policy; hence, a job becomes eligible to be executed only if all previous jobs from the same task have been completed. At any time point, the jobs of the (at most) $m$-highest priority tasks in the ready queue are executed on the processors. Moreover, intra-task parallelism is forbidden; hence, each task may have at most one job executing on at most one processor at any time. Due to the prohibition of intra-task parallelism, we may have more than $m$ jobs available to be executed, but less than $m$ jobs are executed.

Throughout this paper, we refer to the utilization factor of task $\tau_{i}$ as $U_{i}=C_{i} / T_{i}$. We further assume the total utilization $U_{\Sigma}=\sum_{i=1}^{n} U_{i} \leq m$ and $U_{i} \leq 1$. We let $h p(i)$ denote the set of tasks with priority higher than that of task $\tau_{i}$. For the simplicity of presentations, we define the following terms:

- A legal sequence of jobs of the task system $\tau$ : The arrival times of any two consecutive jobs of task $\tau_{i} \in \tau$ are separated by at least $T_{i}$.

- A legal schedule of a legal sequence of jobs: All the 
temporal and scheduling constraints (except the deadlines) are met.

- Worst-case response time $R T_{k}$ of task $\tau_{k}$ : An upper bound of the response times of all the jobs of task $\tau_{k} \in \tau$ under the legal execution for any legal sequence of jobs of $\tau$.

Problem Definition: The objective of this paper is to find the worst-case response time of every task $\tau_{k} \in \tau$ under the given priority assignment. Throughout the paper, we will implicitly only analyze a specific task $\tau_{k}$ under the assumption that the worst-case response time $R T_{i} \leq D_{i}$ of every higher-priority task $\tau_{i}$ in $h p(k)$ has been already analyzed. We will implicitly focus on the cases when $|h p(k)| \geq m$, since the cases with $|h p(k)|<m$ can be trivially solved by $R T_{k} \leq C_{k}$ (under the assumption $C_{k} \leq T_{k}$ ).

\section{PRELIMINARY RESULTS AND DEFI- NITIONS}

This section reviews some results in the literature with respect to the worst-case response-time analysis. We will first explain the level-k busy interval (period) concept by Lehoczky [23] in uniprocessor systems and extend the concept for multiprocessor systems. As our analysis also exploits the workload function, as defined in $[10,15]$, we also provide its definition at the end of this section.

\subsection{Level-k Busy Interval for Task $\tau_{k}$}

For the special case with uniprocessor systems when $m=$ 1 , the response-time analysis for sporadic arbitrary-deadline task systems under fixed-priority scheduling has been developed in [23]. The analysis utilizes a level- $k$ busy interval concept to evaluate the worst-case response time. For such a case, we release all the higher-priority tasks in $h p(k)$ together with task $\tau_{k}$ at time 0 and all the subsequent jobs are released as early as possible by respecting to the minimum inter-arrival time. The level-k busy interval finishes when a job of task $\tau_{k}$ finishes before the next release of a job of task $\tau_{k}$.

For the $h$-th job of task $\tau_{k}$ in the level-k busy interval, the finishing time $R_{k, h}$ is the minimum $t$ such that

$$
h C_{k}+\sum_{\tau_{i} \in h p(k)}\left\lceil\frac{t}{T_{i}}\right\rceil C_{i} \leq t,
$$

and, hence, its response time is $R_{k, h}-(h-1) T_{k}$. The level-k busy interval of task $\tau_{k}$ finishes at the $h$-th job if $R_{k, h} \leq h T_{k}$.

The above analysis works on one processor. However, it requires quite some annotations for the general cases when $m \geq 2$. There are some difficulties for multiprocessor systems. In general, we are unaware of the worst-case release pattern of the higher-priority tasks in $h p(k)$ when $m \geq 2$. The level-k busy interval of task $\tau_{k}$ may have different arrival patterns to create the worst-case response time for different $h$ s. As a result, all possible cases with some unfinished jobs before the arrival of a job have to be considered!

Towards this, we need more precise definitions to capture all the busy intervals for task $\tau_{k}$ and use them to safely bound the worst-case response time. The first definition defines whether a task is busy at time $\theta$ in a legal schedule.

Definition 1 (TAsk Busy). A task is said busy at time instant $\theta$ in a legal schedule if at least one jo $\overline{b \text { of the }}$ task that arrives earlier than $\theta$ has not yet completed its execution at time instant $\theta$.

Note that, by the definition, even if a task is busy at time $\theta$, it may not be executed at time $\theta$. From Definition 2 to Definition 3, we will look at a specific time interval starting from time $\theta_{a}$ in a legal schedule of a legal sequence of jobs of $\tau$.

Definition 2 (A Level-K Busy Interval). A level$k$ busy interval of task $\tau_{k}$ in a legal schedule is an interval $\left[\theta_{a}, \theta_{a}+t\right)$ of time in which, in the legal schedule, (1) task $\tau_{k}$ is busy at all the time points in the interval, and (2) task $\tau_{k}$ is not busy right prior to $\theta_{a}$.

Definition 3 (An $h$-Incomplete Busy Interval). A level-k busy interval $\left[\theta_{a}, \theta_{a}+t\right)$ in a legal schedule is an $h$-incomplete busy interval if the $h$ th-job of task $\tau_{k}$ released in the busy interval $\left[\theta_{a}, \theta_{a}+t\right)$ has not completed yet at time $\theta_{a}+t$.

The above definitions are general forms of the level-k busy interval in [23], i.e., $\theta_{a}$ is 0 for the case when $m$ is 1 . If $\theta_{a}$ is given, the maximum $h$-incomplete busy interval $\left[\theta_{a}, \theta_{a}+t^{*}\right)$ (under the assumption to start from $\theta_{a}$ ) in a legal schedule happens when task $\tau_{k}$ is not busy at time $\theta_{a}+t^{*}$ or task $\tau_{k}$ is $(h+1)$-incomplete at time $\theta_{a}+t^{*}$. Moreover, according to the above definition, for a given $\theta_{a}$ as a fixed left point in the busy intervals, an $(h+1)$-incomplete busy interval (if it exists) completely covers an $h$-incomplete busy interval.

Definition 4 (Maximum $h$-Incomplete Length). The maximum $h$-incomplete length $R_{k, h}$ is the maximum interval length among all the $h$-incomplete busy intervals in the legal schedules of all the legal sequences of the jobs of task system $\tau$.

According to the above definitions, we can reach the following lemma regarding the worst-case response time $R T_{k, h}$ of the $h$ th-job released in a level-k busy interval.

LEMma 1. Suppose that $R_{k, h}$ is known. The worstcase response time $R T_{k, h}$ of the $h$ th-job released in an $h$-incomplete busy interval is at most $R_{k, h}-(h-1) T_{k}$, i.e.,

$$
R T_{k, h} \leq R_{k, h}-(h-1) T_{k}
$$

Proof. According to Definition 4, we know that the length of any $h$-incomplete busy interval of task $\tau_{k}$ is at most $R_{k, h}$. For an $h$-incomplete busy interval, starting at time $\theta_{a}$, this implies that the finishing time of the $h$-th job released no earlier than $\theta_{a}$ is at most $\theta_{a}+R_{k, h}$. In a legal sequence of jobs of task system $\tau$, we also know that the arrival time of the $h$-th job released no earlier than $\theta_{a}$ is at least $\theta_{a}+(h-1) T_{k}$. Therefore, we reach the conclusion.

Based on Lemma 1 , we can now obtain the worst-case response time of task $\tau_{k}$ by evaluating all possible $R T_{k, h}$ :

$$
R T_{k} \leq \max _{h=1,2, \ldots}\left\{R T_{k, h}\right\}
$$

\subsection{Workload Functions}

The concept of workload function has been widely used in real-time schedulability analysis $[10,15]$. Briefly, for a given interval length $t$, the workload function of task $\tau_{i}$ is defined to be the largest accumulative execution time of the legal sequence of the jobs of task $\tau_{i}$ over an interval of length $t$ that could be legally executed within the interval. We provide the formal definition as follows: 
Definition 5 (WorkloAd Function [10,15]). For any interval length $t$, the workload function $W_{i}(t)$ of a sporadic task $\tau_{i}$ bounds the maximum cumulative execution requirement by jobs of $\tau_{i}$ that are released and may execute within any interval of length $t$.

$$
W_{i}(t)=\left\lfloor\frac{t}{T_{i}}\right\rfloor C_{i}+\min \left(t \bmod T_{i}, C_{i}\right)
$$

It has been shown in [15] that the workload function is upper-bounded by the following linear function:

$$
W_{i}(t) \leq U_{i} t+C_{i}\left(1-U_{i}\right)
$$

\section{RESPONSE TIME ANALYSIS}

Based on the observations in Section 3, we will first analyze a safe upper bound of $R_{k, h}$ for a given $h$. Then, the worst-case response time of task $\tau_{k}$ can be obtained by evaluating all possible values of $h$. Although the analysis by Guan et al. [22] is optimistic for arbitrary-deadline task systems, some observations can still be applied. Throughout the section, we will implicitly only analyze a specific task $\tau_{k}$ under the assumption that the worst-case response time $R T_{i} \leq D_{i}$ of every higher-priority task $\tau_{i}$ in $h p(k)$ has been already analyzed.

\subsection{Safe Bound of $R_{k, h}$ for A Given $h$}

We will first shortly review the analysis by Guan et al. [22] and then explain how we plan to conquer the problem to safely derive $R_{k, h}$. Suppose that an $h$-incomplete busy interval of task $\tau_{k}$ begins at time-instant $\theta_{a}$. Suppose that this $h$-incomplete busy interval finishes at time $\theta_{a}+B_{k, h}$. That is, at time $\theta_{a}+B_{k, h}$, the $h$ th-job in this $h$-incomplete busy interval finishes. Throughout this section, we only focus on this interval $\left[\theta_{a}, \theta_{a}+B_{k, h}\right)$ under a legal schedule of a legal sequence of jobs of task system $\tau$.

We first remove some unnecessary jobs in the legal sequence of jobs of task system $\tau$. From this legal sequence of jobs, we first discard all those jobs that have priority lower than task $\tau_{k}$. Since those jobs with priority lower than $\tau_{k}$ have no effect on the scheduling of the jobs generated by $h p(k)$ and task $\tau_{k}$, this removal does not change the scheduling for tasks $\tau_{k}$ and $h p(k)$ in the interval $\left[\theta_{a}, \theta_{a}+\right.$ $\left.B_{k, h}\right)$. Moreover, we also remove the jobs of task $\tau_{k}$ that are released strictly before $\theta_{a}$. Similarly, due to the definition of the $h$-incomplete busy interval, these jobs also have no influence on the scheduling of the jobs generated by $h p(k)$ and task $\tau_{k}$ in the interval $\left[\theta_{a}, \theta_{a}+B_{k, h}\right)$. For the rest of our analysis in this subsection, we will consider only the above reduced legal sequence of jobs.

\subsubsection{Extending the h-Incomplete Busy Interval}

The technique for extending the $h$-incomplete busy interval is needed for precisely bounding the amount of work resulting from higher-priority tasks $\tau_{i}$ in $h p(k)$. Here, we will use the techniques proposed by Baruah [8] and also adopted by Guan et al. [22]. Let $\theta_{0}$ denote the latest timeinstant $\leq \theta_{a}$ at which at least one processor is idle in the legal schedule of the reduced legal sequence of jobs. For notational brevity, let $A_{k, h}=\theta_{a}-\theta_{0}$.

Example: Figure 1 illustrates a 3 -incomplete busy interval $\left[\theta_{a}, \theta_{a}+B_{k, h}\right)$ and a downward extension of the interval to time $\theta_{0}$. Task $\tau_{k}$ is not busy prior to the arrival of the first of these 3 jobs, the first job completes its execution

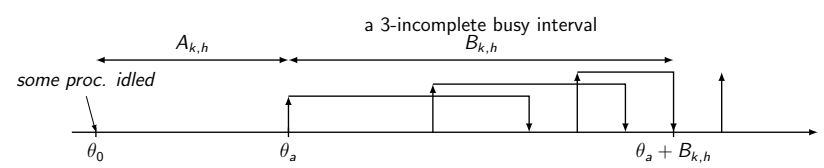

Figure 1: An example of an $h$-incomplete busy interval and the downward extension to $\theta_{0}$.

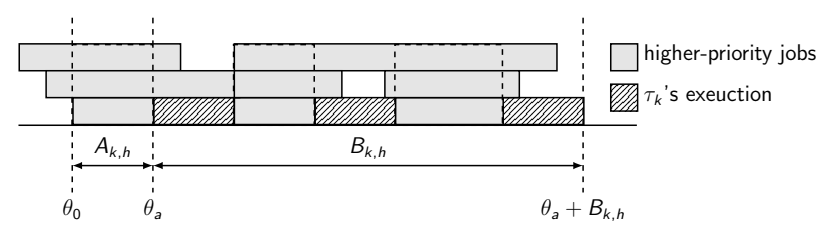

Figure 2: All processors are executing other higher-priority tasks whenever $\tau_{k}$ is not executing. $A_{k, h}=\theta_{a}-\theta_{0}$.

after the second job arrives, and the second job completes its execution after the third job arrives. Thus, the task is continuously busy after the arrival of the first job shown, and $\theta_{a}$ is hence set equal to the arrival time of this job. $\square$

We then have the following observations:

- $\theta_{a}$ is the arrival time of some job of task $\tau_{k}$.

- The contribution from the jobs of task $\tau_{k}$ over $\left[\theta_{0}, \theta_{a}\right)$ is zero due to the reduced legal sequence of jobs.

Another insight on multiprocessors is the lower bound on the workload of the higher-priority tasks in $h p(k)$ in the $h$-incomplete busy interval, as used in most of analysis in the literature $[4,8,22,26]$ : Due to the $h$-incomplete busy interval of task $\tau_{k}$ in the interval $\left[\theta_{a}, \theta_{a}+B_{k, h}\right)$ and the definition of $\theta_{0}$, for any $0<t<B_{k, h}$, the sum of the length in interval $\left[\theta_{0}, \theta_{a}+t\right)$ in which $\tau_{k}$ does not execute must be strictly larger than $A_{k, h}+t-h C_{k}$. The situation is illustrated for $m=3$ processors in Figure 2. The striped rectangles indicate the execution of task $\tau_{k}$ during the $h$ incomplete busy period. The dashed rectangle indicates the intervals in which all $m$ processors execute higher-priority jobs.

Let us denote by $\Gamma_{k}$ a collection of intervals, not necessarily contiguous, of cumulative length $B_{k, h}-h C_{k}$ over $\left[\theta_{a}, \theta_{a}+B_{k, h}\right)$, during which all $m$ processors are executing jobs other than $\tau_{k}$ 's jobs in the legal schedule.

Definition 6 (Higher-Priority Interference). We denote $\Omega\left(A_{k, h}+t\right)$ as the maximum total amount of execution times of the higher-priority interference from the tasks in $h p(k)$ in the time interval $\left[\theta_{0}, \theta_{a}+t\right)$.

From the above observations, an $h$-incomplete busy interval $\left[\theta_{a}, \theta_{a}+B_{k, h}\right)$ of task $\tau_{k}$ requires that

$$
\forall 0<t<B_{k, h}, \Omega\left(A_{k, h}+t\right)>m \times\left(A_{k, h}+t-h C_{k}\right)
$$

Therefore, we can reach the following lemma.

Lemma 2. For a given $A_{k, h}$ and $h$, the maximum $h$ incomplete length $R_{k, h}$ is upper bounded by the minimum value $B_{k, h}$ satisfying the following equation

$$
\Omega\left(A_{k, h}+B_{k, h}\right) \leq m \times\left(B_{k, h}+A_{k, h}-h C_{k}\right)
$$


Note that Lemma 2 can be thought of as a generalized result of Theorem 2 in [8], where $h=1$ and $B_{k, h}=D_{k}$.

\subsubsection{Calculating Higher-Priority Interference}

The treatment up to here is identical to the analysis by Guan et al. in [22]. As long as we can safely bound $\Omega\left(A_{k, h}+\right.$ $\left.B_{k, h}\right)$ for an $h$-incomplete busy interval of task $\tau_{k}$ defined above, we can use Lemma 2 to get $R_{k, h}$. However, calculating $\Omega\left(A_{k, h}+B_{k, h}\right)$ is not trivial. The derivation in [22] was not safe enough.

To derive the contribution of task $\tau_{i}$ in $h p(k)$ to the interference $\Omega\left(A_{k, h}+B_{k, h}\right)$, the contribution from higherpriority task $\tau_{i}$ can be considered by two parts in the interval $\left[\theta_{0}, \theta_{a}+B_{k, h}\right)$ (see Figure 3$):(i)$ carry-in jobs: the jobs (portion) from task $\tau_{i}$ that arrive prior to $\theta_{0}$ and have not yet completed their execution by $\theta_{0}$, and (ii) body jobs: the jobs of $\tau_{i}$ that have the release time within the interval $\left[\theta_{a}, \theta_{a}+B_{k, h}\right)$.

By the definition of $\theta_{0}$, at most $(m-1)$ tasks are with carry-in jobs at time instant $\theta_{0}$. Consequently, Lemma 3 follows immediately:

LEMMA 3 (GUAN ET AL. [22]). There are at most $(m-$ 1) tasks having carry-in jobs in the interval $\left[\theta_{0}, \theta_{a}+B_{k, h}\right]$.

A higher-priority task $\tau_{i}$ in $h p(k)$ can be considered as either with or without carry-in jobs. We now introduce some notations by considering two cases for the amount of execution time executed for a higher-priority task $\tau_{i}$ in the time interval $\left[\theta_{0}, \theta_{a}+B_{k, h}\right]$ in the legal schedule:

1. the maximum work done by task $\tau_{i}$ without carryin jobs over $\left[\theta_{0}, \theta_{a}\right) \cup\left[\theta_{a}, \theta_{a}+B_{k, h}\right)$ is denoted by $I_{i}^{1}\left(A_{k, h}+B_{k, h}\right)$, and

2. the maximum work done by task $\tau_{i}$ with carry-in jobs ${ }^{1}$ over $\left[\theta_{0}, \theta_{a}\right) \cup\left[\theta_{a}, \theta_{a}+B_{k, h}\right)$ is denoted by $I_{i}^{2}\left(A_{k, h}+\right.$ $\left.B_{k, h}\right)$.

Then we explain how to compute a safe interference upper bound on these two functions $I_{i}^{1}\left(A_{k, h}+B_{k, h}\right)$ and $I_{i}^{2}\left(A_{k, h}+\right.$ $\left.B_{k, h}\right)$, provided that $h, A_{k, h}$, and $B_{k, h}$ are given.

Computing $I_{i}^{1}\left(A_{k, h}+B_{k, h}\right)$. First, if a task $\tau_{i}$ contributes no carry-in jobs, then its contribution to the work over $\left[\theta_{0}, \theta_{a}+B_{k, h}\right)$ must arrive no earlier than $\theta_{0}$ and no later than $\theta_{a}+B_{k, h}$, and the total amount of work is bounded from above by the workload function with an interval length of $A_{k, h}+B_{k, h}$. Consequently, $I_{i}^{1}$ can be sufficiently computed by $W_{i}\left(A_{k, h}+B_{k, h}\right)$, as defined in Section 3.2.

Secondly, the total amount of executed work cannot exceed the total length of the interval in $\left[\theta_{0}, \theta_{a}\right) \cup \Gamma_{k}$. The reason behind this is that any work that exceeds the total length of the interval in $\left[\theta_{0}, \theta_{a}\right) \cup \Gamma_{k}$ cannot be parallelly executed with the other work from $\tau_{i}$ that have contributed to $\left[\theta_{0}, \theta_{a}\right) \cup \Gamma_{k}$, in which the total interference by all tasks busily executes on $m$ processors.

Since both are safe upper bounds, we can safely choose the minimum one between them as the maximum interference. Notice that the total length of $\left[\theta_{0}, \theta_{a}\right) \cup \Gamma_{k}$ is equal to $\left(A_{k, h}+\right.$

${ }^{1}$ The assumption by [22] that at most one carry-in job of a task contributes to the interval $\left[\theta_{0}, \theta_{a}+B_{k, h}\right)$ is optimistic, as shown in [29].
$\left.B_{k, h}-h C_{k}\right)$ and " +1 " models the minimum interference that prevents $\tau_{k}$ from executing in the integer time-domain $[9,22]$. Consequently,

$$
\begin{aligned}
& I_{i}^{1}\left(A_{k, h}+B_{k, h}\right)= \\
& \quad \min \left(W_{i}\left(A_{k, h}+B_{k, h}\right), \max \left(0, A_{k, h}+B_{k, h}-h C_{k}+1\right)\right)
\end{aligned}
$$

Computing $I_{i}^{2}\left(A_{k, h}+B_{k, h}\right)$. Now, consider the case that a higher-priority task $\tau_{i}$ contributes carry-in jobs over $\left[\theta_{0}, \theta_{a}+\right.$ $\left.B_{k, h}\right)$. Let $y_{i}$ denote the arrival time of the first job of $\tau_{i}$ released before $\theta_{0}$ and having an absolute deadline (or worst-case completion time) after $\theta_{0}$. Under the assumption that a higher-priority task $\tau_{i}$ has worst-case response time $R T_{i} \leq D_{i}$, the work of task $\tau_{i}$ executed in the interval $\left[\theta_{0}, \theta_{a}+B_{k, h}\right)$ must arrive no earlier than $y_{i}$ and no later than $\theta_{a}+B_{k, h}$. Consequently, the total amount of work $I_{i}^{2}\left(A_{k, h}+B_{k, h}\right)$ is bounded from above by the workload function with an interval length of $\theta_{0}-y_{i}+A_{k, h}+B_{k, h}$. Since $\theta_{0}-y_{i} \leq R T_{i} \leq D_{i}$, consequently, $I_{i}^{2}\left(A_{k, h}+B_{k, h}\right)$ can be sufficiently computed by $W_{i}\left(D_{i}+A_{k, h}+B_{k, h}\right)$. Together with the bounded length of the interval in $\left[\theta_{0}, \theta_{a}\right) \cup \Gamma_{k}$, we therefore have that

$$
\begin{aligned}
& I_{i}^{2}\left(A_{k, h}+B_{k, h}\right)= \\
& \quad \min \left(W_{i}\left(D_{i}+A_{k, h}+B_{k, h}\right), \max \left(0, A_{k, h}+B_{k, h}-h C_{k}+1\right)\right)
\end{aligned}
$$

Moreover, it is not difficult to see that given an upper bound on the worst-case response time $R T_{i}$, the workload function for carry-in task $\tau_{i}$ can be more precisely bounded from above by $W_{i}\left(R T_{i}+A_{k, h}+B_{k, h}\right)$. Hence, a tighter $I_{i}^{2}\left(A_{k, h}+B_{k, h}\right)$ can be computed as follows:

$$
\begin{aligned}
& \hat{I}_{i}^{2}\left(A_{k, h}+B_{k, h}\right)= \\
& \quad \min \left(W_{i}\left(R T_{i}+A_{k, h}+B_{k, h}\right), \max \left(0, A_{k, h}+B_{k, h}-h C_{k}+1\right)\right)
\end{aligned}
$$

For simplifying the analysis, we only use $I_{i}^{2}\left(A_{k, h}+B_{k, h}\right)$ as the maximum work of a higher-priority task $\tau_{i}$ with carry-in jobs in the following presentation.

From the discussion above, we have seen how to sufficiently quantify the interference from these two types of higher-priority tasks. By Lemma 3 , there are at most $(m-1)$ tasks with carry-in jobs at time instant $\theta_{0}$. Hence, there are at most $(m-1)$ tasks contributing to $I_{i}^{2}\left(A_{k, h}+B_{k, h}\right)$, and the remaining $(k-m)$ tasks in $h p(k)$ must contribute to $I_{i}^{1}\left(A_{k, h}+B_{k, h}\right)$.

Computing $\Omega\left(A_{k, h}+B_{k, h}\right)$. With the above settings of $I_{i}^{1}\left(A_{k, h}+B_{k, h}\right)$ and $I_{i}^{2}\left(A_{k, h}+B_{k, h}\right)$, we can now compute $\Omega\left(A_{k, h}+B_{k, h}\right)$. Let us denote by $I_{i}^{D I F F}\left(A_{k, h}+B_{k, h}\right)$ the difference between $I_{i}^{2}\left(A_{k, h}+B_{k, h}\right)$ and $I_{i}^{1}\left(A_{k, h}+B_{k, h}\right)$ :

$$
I_{i}^{D I F F}\left(A_{k, h}+B_{k, h}\right)=I_{i}^{2}\left(A_{k, h}+B_{k, h}\right)-I_{i}^{1}\left(A_{k, h}+B_{k, h}\right) .
$$




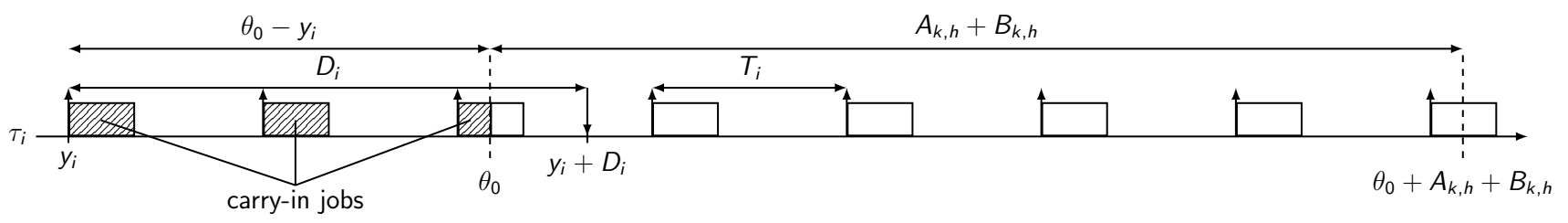

Figure 3: An illustration of releases of a higher-priority task $\tau_{i}$ over $\left[y_{i}, \theta_{a}+B_{k, h}\right)$. The release time of the first job of $\tau_{i}$ released before $\theta_{0}$ and having an absolute deadline (or worst-case completion time) after $\theta_{0}$ is denoted as $y_{i}$.

Therefore, by Lemma 3, we know that

$$
\begin{aligned}
\Omega\left(A_{k, h}+B_{k, h}\right) & =\sum_{\tau_{i} \in h p(k)} I_{i}^{1}\left(A_{k, h}+B_{k, h}\right) \\
+ & \sum_{\substack{(m-1) \text { largest } \\
\tau_{i} \in h p(k)}} I_{i}^{D I F F}\left(A_{k, h}+B_{k, h}\right)
\end{aligned}
$$

where $h p(k)$ denotes the set of tasks that are assigned higher priority than $\tau_{k}$. Note that Eq. (8) can be computed in linear time by using linear-time selection [16] when $A_{k, h}+B_{k, h}$ is given.

\subsection{Compute $R_{k, h}$ using Time Demand Analy- sis}

Without knowing the exact $A_{k, h}$ in the worst-case scenario, intuitively, we have to consider all possible $A_{k, h} \geq 0$ to calculate $R_{k, h}$. Observe that the interference $\Omega\left(A_{k, h}+B_{k, h}\right)$ in Eq. (8) is independent of $A_{k, h}$. Also, $R_{k, h}$ is calculated independently of $A_{k, h}$. Consequently, we only have to consider the case when $A_{k, h}=0$ that results in the maximum $h$-incomplete length $R_{k, h}$. We formally state this with the following lemma:

Lemma 4. By adopting the interference $\Omega\left(A_{k, h}+B_{k, h}\right)$ defined in Eq. (8), the minimum value $B_{k, h}$ by Lemma 2 is maximized when $A_{k, h}=0$. have

Proof. Let $t^{\prime}=A_{k, h}+B_{k, h}$. Equivalently, by Eq (4) we

$$
\Omega\left(t^{\prime}\right) \leq m \times\left(t^{\prime}-h C_{k}\right)
$$

By adopting the interference $\Omega\left(A_{k, h}+B_{k, h}\right)$ defined in Eq. (8), let $X_{k, h}$ be the minimum value of $t^{\prime}$ satisfying Eq. (9). We now consider two separate cases:

- Case 1 with $X_{k, h}<A_{k, h}$ : In this case, we can see that the maximum $h$-incomplete length $R_{k, h}$ is less than the given interval length of $A_{k, h}$. This implies that it is impossible to construct such a case where all $m$ processors busily execute tasks in $h p(k)$ in the time interval $\left[\theta_{0}, \theta_{a}\right]$ with the given interval length of $A_{k, h}$. Hence, we can simply rule out such an $A_{k, h}$.

- Case 2 with $X_{k, h} \geq A_{k, h}$ : We notice that given $h$ and $\tau_{k}, X_{k, h}$ is only dependent on the system parameters, i.e., periods, relative deadlines, and worstcase execution times of higher priority tasks, and is thus unique. Hence, the maximum $h$-incomplete busy interval length $B_{k, h}$ occurs when $A_{k, h}=0$ in our analysis.
In either case we can see that when $A_{k, h}=0$, the minimum $B_{k, h}$ by Lemma 2 is maximized.

By Lemmas 2 and $4, R_{k, h}$ is upper bounded by the smallest $t$ satisfying the following inequality:

$$
\Omega(t) \leq m \times\left(t-h C_{k}\right)
$$

\subsection{Worst-Case Response Time of Task $\tau_{k}$}

By the above analysis, we can now derive the worst-case response time of task $\tau_{k}$ by the following theorem.

THEOREM 1. The worst-case response time $R T_{k}$ of task $\tau_{k}$ is at most:

$$
R T_{k} \leq R T_{k}^{*}=\max _{h \in\{1, \ldots, H\}}\left\{R_{k, h}^{*}-(h-1) T_{k}\right\}
$$

where $H=\min \left\{h \geq 1 \mid \frac{\Omega\left(h T_{i}\right)}{m}+h C_{k} \leq h T_{k}\right\}$, and $R_{k, h}^{*}$ is defined as the minimum $t$ satisfying $E q$. (10).

Proof. Roughly speaking, $H$ represents the maximum $h$ to have $h$-incomplete busy intervals for task $\tau_{k}$. Therefore, by Lemmas 1,2 , and 4 , we reach the conclusion.

Even though $H$ may become infinite, the TDA can be terminated whenever a deadline miss occurs:

$$
\frac{\Omega\left((h-1) T_{k}+D_{k}\right)}{m}+h C_{k}>(h-1) T_{k}+D_{k}
$$

COROllary 1 (TDA). An arbitrary-deadline sporadic task system $\tau$ is schedulable on $m$ identical processors under fixed-priority scheduling if for all tasks $\tau_{k} \in \tau$,

$$
R T_{k}^{*} \leq D_{k}
$$

where $R T_{k}^{*}$ is as defined in Theorem 1.

\section{COMPARISON BETWEEN CARRY-IN WORKLOAD FUNCTIONS}

The carry-in workload function derived in [11,22] shows that the worst-case scenario in constrained-deadline systems occurs when some job of $\tau_{i}$ executing $C_{i}$ units precedes the end of a contiguous interval of length $t$ and the earliest job having absolute deadline within the interval finishes its execution at the end of its deadline (also see Figure 4a). This scenario may accord with the work executing in the schedule as long as the worst-case response time is equal to its relative deadline. On the other hand, our carry-in workload function overly counts the carry-in job that is assumed to arrive $D_{i}$ time-units prior to $t$ (also see Figure 4b), even if such a job cannot effectively execute over the interval. Hence, our carry-in workload function for constrained-deadline tasks is 


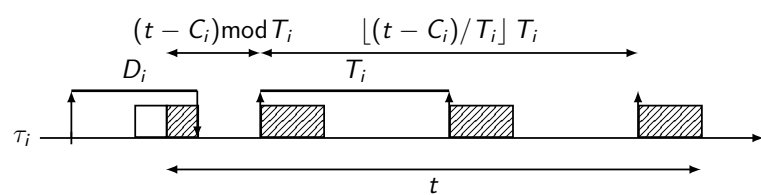

(a) The workload function in $[11,22]$

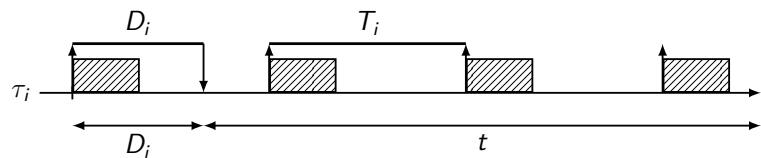

(b) Our workload function

Figure 4: Comparison between the workload function in [11,22] and ours for a carry-in task, assumed $R_{i}=D_{i}$

inferior to that in $[11,22]$ : at most $C_{i}$ may be overestimated. Nevertheless, empirical results show that the difference is insignificant in terms of schedulability, as will be seen later. On the other hand, the assumption that there is at most one carry-in job of a carry-in arbitrary-deadline task is problematic for the carry-in workload function derived in [11,22]. A corresponding counterexample can be found in [29]. Hence, the revised workload function is the first one proved correct for arbitrary-deadline tasks.

\section{LINEAR-TIME UPPER BOUND UNDER FP}

In this section we present a linear-time response time analysis. This test determines the upper bound on the response time under global fixed-priority scheduling upon the values of the tasks' parameters, i.e., the worst-case execution time, the relative deadline, and the period.

We first linearize the upper interference function $\Omega(t)$ by using Eq. (2) in the following lemma:

Lemma 5. For all $t>0$

$$
\Omega(t) \leq\left(Z_{\Sigma}+\sum_{\tau_{i} \in h p(k)}\left(t U_{i}+C_{i}\left(1-U_{i}\right)\right)\right)
$$

where $Z_{\Sigma}$ denotes the sum of the $(m-1)$ largest $U_{i} D_{i}$ 's among the tasks in $h p(k)$.

Proof. Let $S$ denote the set of the $(m-1)$ largest $I_{i}^{D I F F}(t)$.

$$
\begin{aligned}
\Omega(t) & =\sum_{\tau_{i} \in h p(k)} I_{i}^{1}(t)+\sum_{\text {the }(m-1) \text { largest }} I_{i}^{D I F F}(t) \\
& =\sum_{\tau_{i} \in h p(k) \backslash S} I_{i}^{1}(t)+\sum_{\tau_{i} \in S} I_{i}^{2}(t) \\
& \leq \sum_{\tau_{i} \in h p(k) \backslash S} W_{i}(t)+\sum_{\tau_{i} \in S} W_{i}\left(t+D_{i}\right) \\
& \stackrel{(2)}{\leq} Z_{\Sigma}+\sum_{\tau_{i} \in h p(k)}\left(t U_{i}+C_{i}\left(1-U_{i}\right)\right)
\end{aligned}
$$

Subsequently, the following lemma provides an upper bound on the maximum length of the $h$-incomplete busy periods of task $\tau_{k}$.

Lemma 6.

$$
R_{k, h} \leq R_{k, h}^{\dagger} \stackrel{\text { def }}{=} \frac{h m C_{k}+Z_{\Sigma}+\sum_{\tau_{i} \in h p(k)} C_{i}\left(1-U_{i}\right)}{m-\sum_{\tau_{i} \in h p(k)} U_{i}}
$$

where $Z_{\Sigma}$ denotes the sum of the $(m-1)$ largest $D_{i} U_{i}$ 's.
Proof. Substituting $\Omega(t)$ in the recurrence Eq. (10) by the one in Lemma 5 , we can find the point $t$ of the intersection, which leads to the statement.

By Lemmas 1 and $6, R T_{k, h}$ is bounded from above by

$$
R T_{k, h} \leq R T_{k, h}^{\dagger} \stackrel{\text { def }}{=} R_{k, h}^{\dagger}-(h-1) T_{k}
$$

where $R_{k, h}^{\dagger}$ is defined in Lemma 6 . Intuitively, one may examine all possible $h$ in the above equation to ensure that the upper bound on the worst-case response time. Our observation is that $R T_{k, h}^{\dagger}$ defined by Eq. (15) is monotonically decreasing with respect to $h$, under certain condition. We state this with the following lemma:

LEMMA 7. Suppose that

$$
m U_{k}+\sum_{\tau_{i} \in h p(k)} U_{i}<m
$$

Then, for any integer $h \geq 1$,

$$
R T_{k, 1}^{\dagger} \geq R T_{k, h}^{\dagger}
$$

where $R T_{k, h}^{\dagger}$ is as defined in Eq. (15).

Proof. We now prove that $R_{k, h}^{\dagger}-(h-1) T_{k}$ is maximized when $h$ is 1 , under Condition (16). Differentiating $R_{k, h}^{\dagger}-$ $(h-1) T_{k}$ with respect to $h$, we have that

$$
\begin{aligned}
\frac{\partial}{\partial h}\left(R_{k, h}^{\dagger}-(h-1) T_{k}\right) & =\frac{m C_{k}}{m-\sum_{\tau_{i} \in h p(k)} U_{i}}-T_{k} \\
& =T_{k}\left(\frac{m U_{k}}{m-\sum_{\tau_{i} \in h p(k)} U_{i}}-1\right) \\
& =T_{k}\left(\frac{m U_{k}+\sum_{\tau_{i} \in h p(k)} U_{i}-m}{m-\sum_{\tau_{i} \in h p(k)} U_{i}}\right)
\end{aligned}
$$

which is negative due to our assumption $m U_{k}+\sum_{\tau_{i} \in h p(k)} U_{i}<$ $m$. Since $R_{k, h}^{\dagger}-(h-1) T_{k}$ is decreasing with respect to $h$, the maximum occurs when $h=1$.

Putting these pieces together, Theorem 2 provides the response time upper bound of arbitrary-deadline tasks on multiprocessor systems.

THEOREM 2. The worst-case response time $R T_{k}$ of task $\tau_{k}$ is at most

$$
R T_{k} \leq R T_{k}^{\dagger}= \begin{cases}R_{k}^{u p} & \text { if } m U_{k}+\sum_{\tau_{i} \in h p(k)} U_{i}<m \\ \infty & \text { otherwise. }\end{cases}
$$

where $R_{k}^{u p}=\frac{m C_{k}+Z_{\Sigma}+\sum_{\tau_{i} \in h p(k)} C_{i}\left(1-U_{i}\right)}{m-\sum_{\tau_{i} \in h p(k)} U_{i}}$ and $Z_{\Sigma}$ denotes the sum of the $(m-1)$ largest $D_{i} U_{i}$ 's among the tasks in $h p(k)$. 
Proof. By Lemmas 6 and 7, we can conclude this theorem by setting $h=1$ in Eq. (15).

Subsequently, we provide our linear-time upper bound (LTUB) on the worst-case response time in the follow corollary:

Corollary 2 (LTUB). An arbitrary-deadline sporadic task system $\tau$ is schedulable on $m$ identical processors under fixed-priority scheduling if for all tasks $\tau_{k} \in \tau$,

$$
R T_{k}^{\dagger} \leq D_{k}
$$

where $R T_{k}^{\dagger}$ is as defined in Theorem 2.

\section{EXPERIMENTS}

In this section, we conduct extensive experiments using synthesized task sets for evaluating the proposed tests. The metric to compare results is to measure the acceptance ratio of the above tests with respect to a given goal of task set utilization. We generate 100 task sets for each utilization level, from 0.01 to 0.99 , in steps of 0.01 . For brevity, the tickers were set every three steps. The acceptance ratio of a level is said to be the number of task sets that are schedulable divided by the number of task sets for this level, i.e., 100 .

\subsection{Simulation Environment}

We first generated a set of sporadic tasks. The cardinality of the task set was 5 times the number of processors. The UUniFast-Discard method [14] was adopted to generate a set of utilization values with the given goal. We here used the approach suggested by Davis and Burns [20] to generate the task period according to the exponential distribution. The order of magnitude $p$ to control the period values between largest and smallest periods is parameterized in evaluations. (E.g., $1-10 m s$ for $p=1,1-100 m s$ for $p=2$, etc.). We evaluate these tests in 8 multiprocessor systems with $p \in[1,2,3]$. Similar results can be seen in different numbers of processors. The priority of task is assigned according to deadline-monotonic (DM) scheduling: the smaller the relative deadline, the higher the priority level.

We note that deadline-monotonic (DM) scheduling is not optimal on multiprocessor systems. Alternatively, one can use the laxity-monotonic (LM) scheduling: the smaller the laxity $D_{i}-C_{i}$, the higher the priority level [2]. Also, it is not difficult to see that the proposed TDA analysis and the linear-time response bound comply with the required conditions for the optimal priority assignment (OPA) compatibility provided in [18]. Hence, our proposed tests are compatible with OPA: if there exists feasible priority assignments by our test, OPA returns one of them. It is also worth noting that Davis and Burns have suggested that being OPA-compatible is as important as being effective since priority assignment is an important factor in determining the schedulability of tasksets under global fixed priority pre-emptive scheduling [18]. Consequently, applying the tighter test but not OPA-comptiable, e.g. maximum work function $\hat{I}_{i}^{2}\left(A_{k, h}+B_{k, h}\right)$, by using DM, may not yield better performance than the less effective but OPA-comptiable test using OPA priority assignment. Nevertheless, we here focus on showing the effectiveness of the tests themselves, and similar results can be seen under different priority assignment policies.

\subsection{Constrained-Deadline Systems \& Results}

The execution time was set accordingly, i.e., $C_{i}=T_{i} U_{i}$. Task relative deadlines were uniformly drawn from the interval $\left[0.8 T_{i}, T_{i}\right]$.

The tests evaluated are shown as follows:

- BCL: the polynomial-time test in Theorem 4 in [11].

- FF: the force-forward (FF) analysis in Eq. (5) in [6].

- $B A K$ : the $O\left(n^{3}\right)$ test in Theorem 11 in [4].

- Guan: the TDA analysis by Guan [22].

- TDA: Corollary 1 in this paper.

- LTUB: Corollary 2 in this paper.

Results. Figure 5 presents the evaluation result in constraineddeadline systems. We first notice that the response time analysis with limited carry-in tasks, namely Guan and TDA, can admit the most number of task sets. Overall, these two tests achieve similar performance, even though our TDA is inferior than Guan due to the over-approximate workload function, as mentioned earlier. As shown in Figure 5, BCL, $F F$, and $B A K$ perform poorer than $L T U B$, even though their computational complexity is higher than $L T U B$. (pseudopolynomial time for $F F$ and $O\left(n^{3}\right)$ for $\left.B A K\right)$. It is noticeable that $T D A$ is only slightly advantageous to $L T U B$ in case of $p=2$ and $p=3$ (Figure 5(b) and (c)) where the TDA essentially suffers from its high computational complexity. Hence, in practice, one would expect that the TDA be adopted in the low order of magnitude of task periods and the linear-time response time bound in the high order of the magnitude.

\subsection{Arbitrary-Deadline Systems \& Results}

The execution time was set accordingly, i.e., $C_{i}=T_{i} U_{i}$. Task relative deadlines were uniformly drawn from the interval $\left[0.8 T_{i}, 2 T_{i}\right]$.

The tests evaluated are shown as follows:

- $L O A D$ : the load-based analysis [7].

- $B A K$ : the $O\left(n^{3}\right)$ test in Theorem 11 [4].

- LTUB: Corollary 2 in this paper.

- TDA: Corollary 1 in this paper.

Results. Figure 6 presents the result in arbitrary-deadline systems where $\frac{D_{i}}{T_{i}} \in[0.8,2]$. Due to the overly optimistic workload function, Guan's analysis becomes unsafe for arbitrary-deadline tasks. The proposed TDA is the best TDA with limited carry-in interferences in arbitrarydeadline systems, as can be seen in Figure 6. Also, in terms of schedulability and time complexity, the proposed lineartime upper bound $L T U B$ is superior to $L O A D$ and $B A K$, where the time complexity is pseudo-polynomial for $L O A D$ and $O\left(n^{3}\right)$ for $B A K$.

\section{CONCLUSIONS}

In this work, we propose the response time analysis for sporadic arbitrary-deadline tasks on multiprocessor systems under fixed-priority scheduling. Further, we derive a lineartime response time bound that provides an amenable solution for the the admission control and the interactive system 


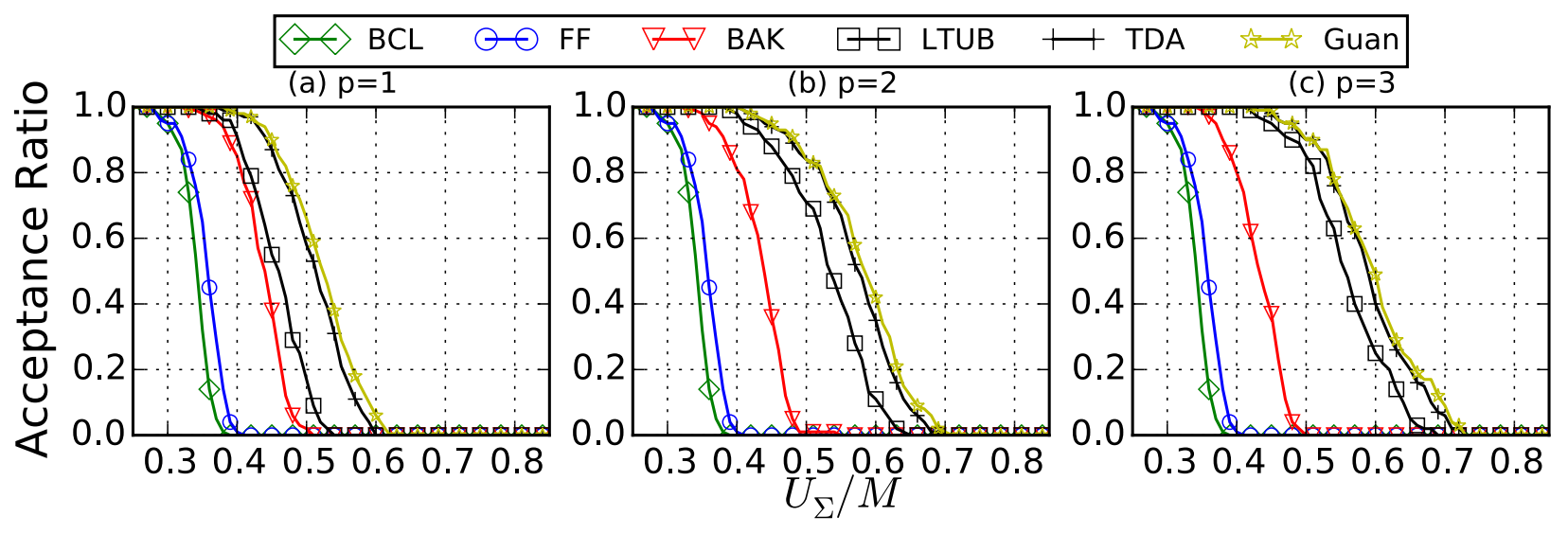

Figure 5: Acceptance ratio comparison with different $p$ on 8-multiprocessor, constrained-deadline systems where $\frac{D_{i}}{T_{i}} \in[0.8,1]$.

and prototyping. To the best of our knowledge, this is the first work presenting a linear-time response time bound on multiprocessor systems. We further conclude that the lineartime response time bound yields high performance compared to the state-of-the-art TDA tests, especially in case of large task periods, where time-demand analysis tests suffer from their computational complexity.

\section{ACKNOWLEDGMENTS}

This paper is supported by DFG, as part of the Collaborative Research Center SFB876 (http://sfb876.tu-dortmund.de/).

\section{REFERENCES}

[1] B. Andersson, S. Baruah, and J. Jonsson. Static-priority scheduling on multiprocessors. In Real-Time Systems Symposium (RTSS 2001), pages 193-202, 2001.

[2] B. Andersson and J. Jonsson. Fixed-priority preemptive multiprocessor scheduling: to partition or not to partition. In Real-Time Computing Systems and Applications, 2000. Proceedings. Seventh International Conference on, pages 337-346. IEEE, 2000.

[3] T. P. Baker. Multiprocessor edf and deadline monotonic schedulability analysis. In IEEE Real-Time Systems Symposium, pages 120-129, 2003.

[4] T. P. Baker. An analysis of fixed-priority schedulability on a multiprocessor. Real-Time Systems, 32(1-2):49-71, 2006.

[5] T. P. Baker and M. Cirinei. Brute-force determination of multiprocessor schedulability for sets of sporadic hard-deadline tasks. In Principles of Distributed Systems, 11th International Conference, OPODIS 2007, Guadeloupe, French West Indies, December 17-20, 2007. Proceedings, pages 62-75, 2007.

[6] S. Baruah, V. Bonifaci, A. Marchetti-Spaccamela, and S. Stiller. Improved multiprocessor global schedulability analysis. Real-Time Systems, 46(1):3-24, 2010.

[7] S. Baruah and N. Fisher. Global fixed-priority scheduling of arbitrary-deadline sporadic task systems. In Distributed Computing and Networking, pages 215-226. Springer, 2008.
[8] S. K. Baruah. Techniques for multiprocessor global schedulability analysis. In Proceedings of the 28th IEEE Real-Time Systems Symposium, pages 119-128, 2007.

[9] M. Bertogna and M. Cirinei. Response-time analysis for globally scheduled symmetric multiprocessor platforms. In Real-Time Systems Symposium, pages 149-160, 2007.

[10] M. Bertogna, M. Cirinei, and G. Lipari. Improved schedulability analysis of edf on multiprocessor platforms. In Euromicro Conference on Real-Time Systems (ECRTS), pages 209-218, 2005.

[11] M. Bertogna, M. Cirinei, and G. Lipari. New schedulability tests for real-time task sets scheduled by deadline monotonic on multiprocessors. In Principles of Distributed Systems, pages 306-321. Springer, 2006.

[12] E. Bini. The quadratic utilization upper bound for arbitrary deadline real-time tasks. Computers, IEEE Transactions on, 64(2):593-599, 2015.

[13] E. Bini and G. C. Buttazzo. Schedulability analysis of periodic fixed priority systems. Computers, IEEE Transactions on, 53(11):1462-1473, 2004.

[14] E. Bini and G. C. Buttazzo. Measuring the performance of schedulability tests. Real-Time Systems, 30(1-2):129-154, 2005.

[15] E. Bini, T. H. C. Nguyen, P. Richard, and S. K. Baruah. A response-time bound in fixed-priority scheduling with arbitrary deadlines. IEEE Transactions on Computers, 58(2):279, 2009.

[16] M. Blum, R. W. Floyd, V. Pratt, R. L. Rivest, and R. E. Tarjan. Time bounds for selection. Journal of computer and system sciences, 7(4):448-461, 1973.

[17] R. I. Davis and A. Burns. Response time upper bounds for fixed priority real-time systems. In Real-Time Systems Symposium, 2008, pages 407-418. IEEE, 2008.

[18] R. I. Davis and A. Burns. Improved priority assignment for global fixed priority pre-emptive scheduling in multiprocessor real-time systems. Real-Time Systems, 47(1):1-40, 2011.

[19] R. I. Davis and A. Burns. A survey of hard real-time 


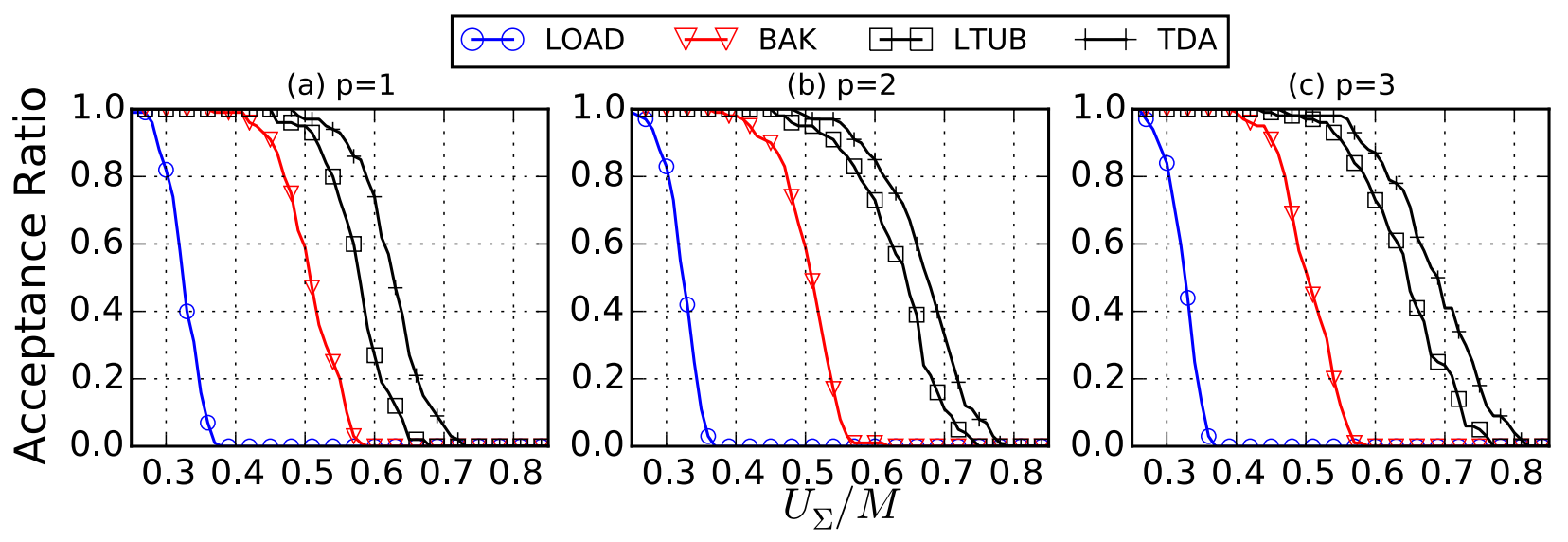

Figure 6: Acceptance ratio comparison with different $p$ on 8-multiprocessor, arbitrary-deadline systems where $\frac{D_{i}}{T_{i}} \in[0.8,2]$.

scheduling for multiprocessor systems. $A C M$ Computing Surveys (CSUR), 43(4):35, 2011.

[20] R. I. Davis, A. Zabos, and A. Burns. Efficient exact schedulability tests for fixed priority real-time systems. Computers, IEEE Transactions on, 57(9):1261-1276, 2008.

[21] G. Geeraerts, J. Goossens, and M. Lindström. Multiprocessor schedulability of arbitrary-deadline sporadic tasks: complexity and antichain algorithm. Real-Time Systems, 49(2):171-218, 2013.

[22] N. Guan, M. Stigge, W. Yi, and G. Yu. New response time bounds for fixed priority multiprocessor scheduling. In Real-Time Systems Symposium, 2009, RTSS 2009. 30th IEEE, pages 387-397. IEEE, 2009.

[23] J. P. Lehoczky. Fixed priority scheduling of periodic task sets with arbitrary deadlines. In Real Time Systems Symposium, pages 201-209, 1990.

[24] C. L. Liu and J. W. Layland. Scheduling algorithms for multiprogramming in a hard-real-time environment. Journal of the ACM (JACM), 20(1):46-61, 1973.

[25] A. K. Mok. Fundamental design problems of distributed systems for the hard-real-time environment. 1983.

[26] C. A. Phillips, C. Stein, E. Torng, and J. Wein. Optimal time-critical scheduling via resource augmentation. In Proceedings of the twenty-ninth annual ACM symposium on Theory of computing, pages 140-149. ACM, 1997.

[27] M. Sjodin and H. Hansson. Improved response-time analysis calculations. In Real-Time Systems Symposium, 1998. Proceedings., The 19th IEEE, pages 399-408. IEEE, 1998.

[28] Y. Sun and G. Lipari. A weak simulation relation for real-time schedulability analysis of global fixed priority scheduling using linear hybrid automata. In 22nd International Conference on Real-Time Networks and Systems, RTNS '14, Versaille, France, October 8-10, 2014, page 35, 2014.

[29] Y. Sun, G. Lipari, N. AGuan, W. Yi, et al. Improving the response time analysis of global fixed-priority multiprocessor scheduling. In IEEE International Conference on Embedded and Real-Time Computing Systems and Applications (RTCSA), pages 1-9, 2014.

[30] K. Tindell, A. Burns, and A. J. Wellings. An extendible approach for analyzing fixed priority hard real-time tasks. Real-Time Systems, 6(2):133-151, 1994. 